\title{
The future SIDIS measurement on transversely polarized deuterons by the COMPASS Collaboration
}

\author{
Franco Bradamante* \\ INFN, Trieste, Italy \\ E-mail: Franco.Bradamante@ts.infn.it
}

\section{on behalf of the COMPASS Collaboration}

Since 2005, measurements of Collins and Sivers asymmetries from the HERMES and COMPASS experiments have allowed to assess that the transversity and the Sivers PDFs are different from zero and measurable in semi-inclusive DIS on transversely polarised targets. Most of the data were collected on proton targets, only small event samples were collected in the early phase of the COMPASS experiment on a deuteron $\left({ }^{6} \mathrm{LiD}\right)$ target and more recently at JLab, on ${ }^{3} \mathrm{He}$, so that the $d$-quark and the sea-quarks PDFs are much more poorly known than the $u$-quark PDFs. This constitutes an important limitation to the knowledge of the transverse spin structure of the nucleon. For this reason the COMPASS Collaboration has proposed to measure semi-inclusive DIS on transversely polarised deuterons with good accuracy, comparable with that of the existing transverse spin asymmetry data on protons. The proposal has been accepted by CERN and the experiment will run in 2021, as soon as the Long Shut-down 2 is over, providing measurements which will stay unique for many years to come. Projections will be given for the extraction of the transversity PDFs, and for the evaluation of the isovector tensor charge of the nucleon.

23rd International Spin Physics Symposium - SPIN2018 -

10-14 September, 2018

Ferrara, Italy

\footnotetext{
* Speaker.
} 


\section{Introduction}

The description of the nucleon structure in hard inclusive processes in terms of collinear parton distribution functions (PDFs) has recently been generalised to take into account the transverse momentum $k_{T}$ of the parton with respect to the nucleon direction. At leading twist a complete picture of the nucleon requires a total of eight transverse-momentum-dependent (TMD) distributions, which depend on the parton light-cone momentum fraction $x$, on a characteristic hard scale $Q^{2}$, and on $k_{T}$ (for reviews, see [1-3]).

When the target nucleon is transversely polarised, eight different spin-dependent azimuthal modulations are expected in the SIDIS cross-section, so that from a measurement of the crosssection and knowing the fragmentation functions (FFs) important information on the TMD PDFs can be extracted. In this domain the HERMES [4,5] and the COMPASS [6-9] Collaborations have performed pioneering measurements at different beam energies $(27$ and $160 \mathrm{GeV} / c$ respectively) and shown beyond any doubt that two spin-dependent azimuthal modulations of the produced hadrons, of the type $\left[1+A_{C} \sin \left(\phi_{h}+\phi_{S}-\pi\right)\right]$ and $\left[1+A_{S} \sin \left(\phi_{h}-\phi_{S}\right)\right]$, are clearly different from zero. The azimuthal angles of the produced hadron transverse momentum and of the spin direction of the target nucleon are defined with respect to the lepton scattering plane, in a reference system in which the $\mathrm{z}$ axis is the virtual-photon direction. The first modulation, called Collins asymmetry, is described as a convolution of the transversity function and a newly introduced TMD FF, the Collins FF, $H_{1}^{\perp}$. The second modulation, called the Sivers asymmetry, is described as a convolution between the Sivers function, a new TMD PDF, and the usual fragmentation function. For all details I refer again to the existing literature [1-3]). The fact that the Collins asymmetry and the Sivers asymmetry have been shown to be non zero (at least on a proton target) implies that:

- the Collins FF $H_{1}^{\perp}$ is different from zero

- the transversity PDF, $h_{1}$, is different from zero

- the Sivers PDF, $f_{1 T}^{\perp}$, is different from zero.

Independent evidence that the Collins FF is different from zero was provided by the measurement of azimuthal asymmetries in inclusive production of hadron pairs in $e^{+} e^{-}$annihilation by the Belle Collaboration [10]. When combining the HERMES [4] and the COMPASS [6-8] data with the Belle measurements of $e^{+} e^{-} \rightarrow$ hadrons, first extractions of the Collins FF and of the transversity PDF have been possible. Some recent extractions of the transversity function, from Ref. [11] and from Ref. [12], are shown in Fig. 1. In spite of the large uncertainties, it is an important achievement. The trend of the functions is similar to that of the $u$ and $d$ helicity distributions, but clearly we are still a long way from being able to assess the difference between the transversity and the helicity PDFs, which is the ultimate goal of this investigation. A second feature in Fig. 1 is that the uncertainty in $h_{1}^{q}$ for the $d$ quark is much larger than in $h_{1}^{q}$ for the $u$ quark. This is due to the fact that most of the existing data have been collected on proton targets, and only few measurements exist on neutron targets. COMPASS has measured transverse spin asymmetries using both proton and deuteron targets. On the deuteron, the asymmetries are compatible with zero [6], but the accuracy of the measurements is definitely inferior to that of the proton data. More recently data have been collected at much lower energy at JLab on a ${ }^{3} \mathrm{He}$ target, essentially a transversely 
polarised neutron target: the measured asymmetries $[13,14]$ are also compatible with zero, but the error bars are again fairly large.

The measurement of the transversity distributions is particularly important because they provide access to the tensor charges $\delta q$, or $g_{T}^{q}$, which are given by the integral

$$
\delta q\left(Q^{2}\right)=\int_{0}^{1} d x\left[h_{1}^{q}\left(x, Q^{2}\right)-h_{1}^{\bar{q}}\left(x, Q^{2}\right)\right]=\int_{0}^{1} d x\left[h_{1}^{q_{v}}\left(x, Q^{2}\right)\right]
$$

In a non-relativistic quark model, $h_{1}^{q}$ is equal to $g_{1}^{q}$, the helicity distribution, and $\delta q$ is equal to the valence quark $q_{v}$ contribution to the nucleon spin. The difference between $h_{1}^{q}$ and $g_{1}^{q}$ provides important constraints to any model of the nucleon. Knowing the quark tensor charges one can construct the isovector nucleon tensor charge $g_{T}=\delta u-\delta d$, a fundamental property of the nucleon which, together with the vector and axial charges, characterizes the nucleon as a whole. Since many years the tensor charge is being calculated with steadily increasing accuracy by lattice QCD [15]. More recently, its connection with possible novel tensor interactions at the $\mathrm{TeV}$ scale in neutron and nuclear $\beta$-decays and its possible contribution to the neutron electric dipole moment (EDM) have also been investigated [16], and possible constraints on new physics beyond the standard model have also been derived [17].

A good knowledge of the tensor charge requires measurements of similar accuracy for deuteron and proton data. For this reason we have proposed [18] to CERN a new one-year (150 days) measurement, scattering the M2 muon beam with $160 \mathrm{GeV} / \mathrm{c}$ momentum on a transversely polarised deuteron target, as soon as the second long-shut down of the CERN accelerator complex (LS2) will be over, using the COMPASS spectrometer in the configuration we have used it in 2010. In the future, JLab12 will provide new and hopefully precise data on both the proton and the deuteron, but at fairly large $x$-values, namely $x>0.05$. The main objective of our proposal is to measure from $x \simeq 0.3$ down to 0.003 and at larger $Q^{2}$ (up to $Q^{2} \simeq 100(\mathrm{GeV} / \mathrm{c})^{2}$ ), thus our measurement will be complementary to the JLab12 measurement and essential both to evaluate the tensor charges and to access the PDFs of the sea quarks.

Our proposal has been accepted by CERN, and the experiment should run in 2021.
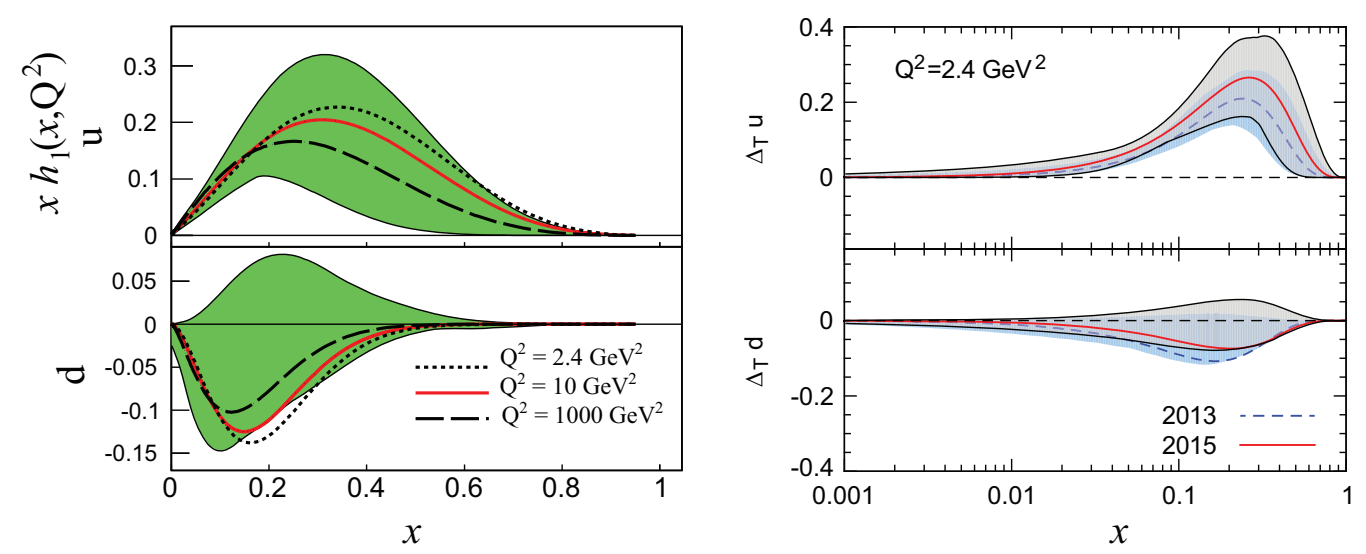

Figure 1: The $u$ and $d$ quark transversity PDFs from recent global fits. The plots are from Ref. [11] (left) and from Ref. [12] (right). Although the notation for the transversity PDF in the plot at the right is not the one used in this paper, the same function is shown in both plots. 


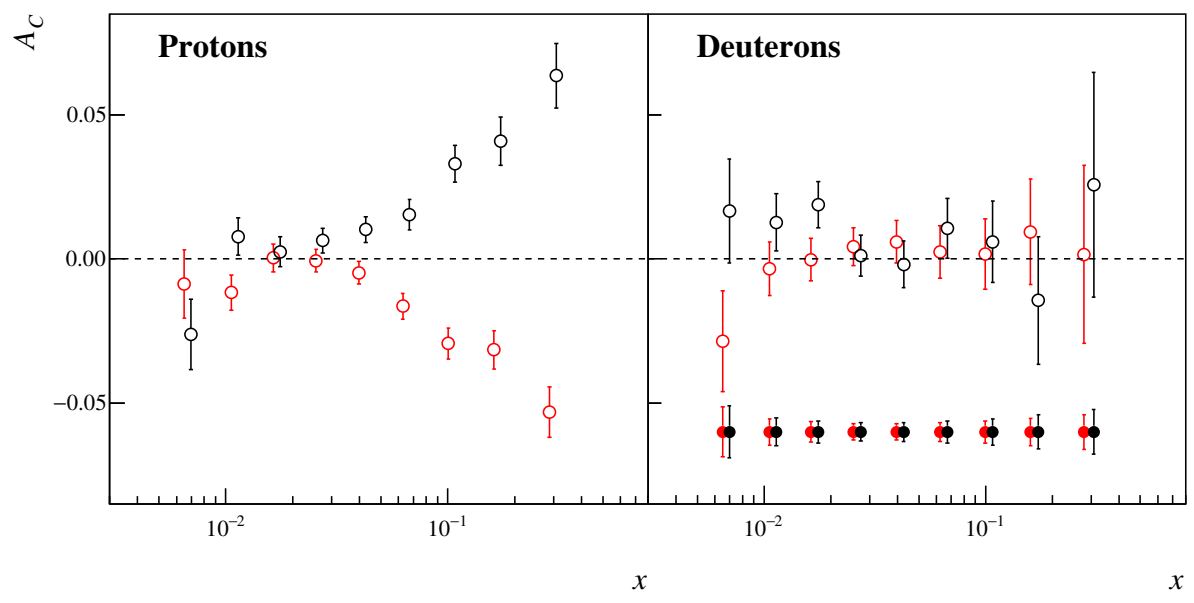

Figure 2: The Collins asymmetry $A_{C}$ obtained from the 2010 data with the polarised proton $\mathrm{NH}_{3}$ target as a function of $x$ (left plot) compared to the results obtained from the runs of 2002, 2003 and 2004 with polarised deuteron ${ }^{6} \mathrm{LiD}$ target (right plot). The red (black) points refer to positive (negative) hadrons. The full points at $A_{C}=-0.06$ in the right plot show the extrapolated statistical errors from the proposed deuteron run.

In the following, for reasons of space and time, I will detail only the case for transversity, but it is clear that the new SIDIS asymmetry data, combined with the good precision HERMES and COMPASS proton data, and with the future high precision JLab12 data, will allow also all the other $u$ and $d$ distribution functions to be extracted with comparable accuracy. This is particularly important for the Sivers distribution. The future EIC will possibly supersede the existing and the proposed measurements, but the new COMPASS contribution will stay there for several years.

\section{Present and extrapolated uncertainties for COMPASS transverse spin asymmetries}

In order to estimate the impact of a future run on a transversely polarised deuteron target, it is convenient to look at Fig. 2, where the Collins asymmetries for positive and negative hadrons obtained from the 2010 data [8] collected using $\mathrm{NH}_{3}$ as a polarised proton target, are shown as a function of $x$ (left panel) and compared to the results obtained from the deuteron runs of 2002, 2003, and 2004 [6], when as target we used ${ }^{6} \mathrm{LiD}$ (right panel). Clearly the accuracy of the data is considerably better for the proton target. At small $x$ the uncertainties are similar, but as a function of $x$, while the Collins asymmetries get larger, the statistical uncertainties of the deuteron asymmetries become larger and larger than those of the proton ones. As shown in Fig. 3, where the ratio of the statistical uncertainties of the asymmetries are plotted as function of $x$, the ratio is larger than a factor of three in the last measured $x$-bin. The reason for this increase with $x$ is the fact that when the deuteron target data were collected, due to the late delivery of the COMPASS polarised target (PT) magnet we used the existing PT magnet previously constructed and used by the SMC Collaboration. The COMPASS PT magnet was operational only in 2005, and we could use it for our transversely polarized proton runs of 2007 and 2010. There is indeed a huge difference between the acceptance of the COMPASS PT magnet and the SMC PT magnet: the COMPASS magnet has a polar angle acceptance of $180 \mathrm{mrad}$ (as seen from the upstream end of the target) while the SMC 


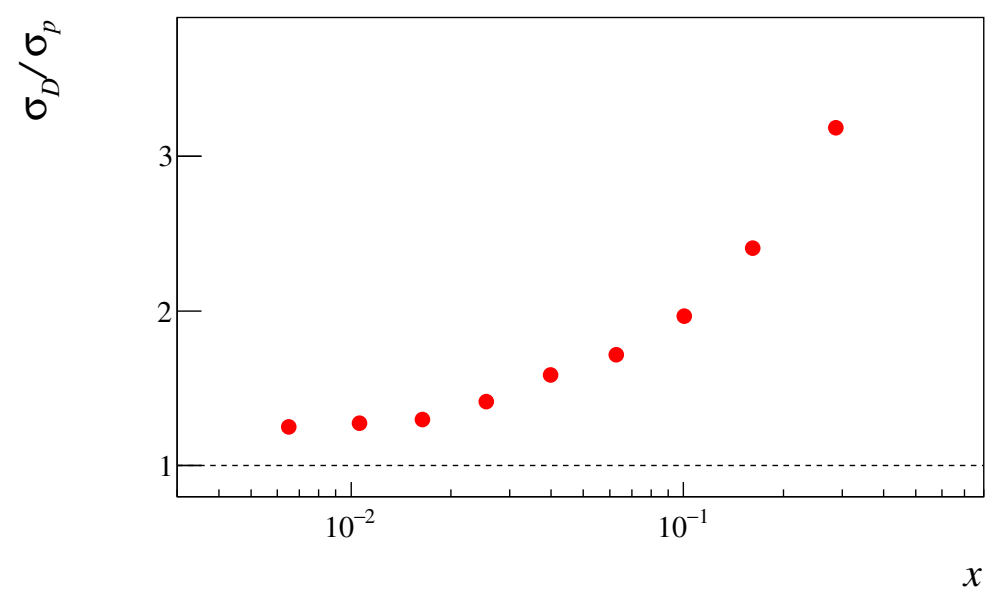

Figure 3: Ratio of the statistical uncertainties of deuteron and proton asymmetries for charged hadrons as measured by COMPASS.

magnet has a corresponding acceptance of $70 \mathrm{mrad}$. A reduced acceptance in scattering angle directly translates into a reduced acceptance at large $x$-Bjorken.

By using the COMPASS PT magnet for the future deuteron run, in one year of data taking in the conditions of the 2010 proton run we can safely expect the number of reconstructed SIDIS events to be the same as in 2010, since target material densities and packing factors are almost identical for ${ }^{6} \mathrm{LiD}$ and $\mathrm{NH}_{3}$. It is then very simple to estimate the statistical errors of the measured Collins asymmetries in the future deuteron run, they will just be the statistical uncertainties of the 2010 proton data scaled by the ratio of the figures of merit of the two targets, namely

$$
r=\frac{F O M_{p}}{F O M_{d}}=\frac{0.155 \cdot 0.80}{0.40 \cdot 0.50}=0.62,
$$

where $F O M(=f P)$ is the figure of merit of the polarized target, $f$ is the dilution factor of the target material, and $P$ is the proton or deuteron polarization. Under the assumption that the spectrometer acceptance and efficiency be the same for the deuteron runs as they were for the 2010 proton run, the statistical errors of the transverse spin asymmetries will be smaller by a factor of 0.62 than those of the proton. The projected errors for the deuteron Collins asymmetries are shown as closed points in the right plot of Fig. 2. We neglect the systematic errors which were estimated to be at most 0.5 times the statistical errors in the 2010 data, so that they increase the total error by less than $10 \%$.

Most important, very much as for the Collins asymmetry, all the target transverse spin asymmetries (TSA) are expected to be measured with a statistical uncertainty equal to 0.62 times the statistical uncertainties of the corresponding asymmetries measured in the 2010 proton run which in all the following we use as a reference.

\section{Present and extrapolated uncertainties for transversity}

In order to quantify the gain in statistical uncertainty in $h_{1}^{u_{v}}$ and $h_{1}^{d_{v}}$, we have extracted twice the $u$ - and $d$-quark transversity, the first time using the existing COMPASS deuteron data, and a 
second time using the projections of Fig. 2 for the statistical errors of the new deuteron data. In both cases all the existing proton data from HERMES and COMPASS have been used. To carry through this evaluation we have followed the procedure of Ref. [19], a point by point extraction of transversity directly from the measured SIDIS and $e^{+} e^{-} \rightarrow$ hadrons asymmetries.

The big advantage of this method is that

- it avoids the use of parametrizations for the unknown functions which introduce systematic errors difficult to estimate

- the transversity PDFs, their statistical uncertainties and their correlation coefficients can all be calculated algebraically from the measured asymmetries

- it allows to extract separately the valence quark transversity and the sea quark transversity as different linear combinations of the four measured spin asymmetries (positive/negative pions on proton/deuteron targets)

but

- it requires asymmetry measurements on different targets ( $\mathrm{p}, \mathrm{d}$ or $\mathrm{n}$ ) available at the same $x$-values and in the same kinematic ranges.

For what concerns the last point, there has been no problem to add the results from the COMPASS proton data collected in 2007 [7]: the data were taken with the same experimental apparatus, at the same beam energy, have been binned in the same $x$-intervals, and we had already merged them in the past and put the 2007 and 2010 combined results in HepData [20]. However, merging the HERMES results with ours needs an approximation. For $x>0.032$ the HERMES range overlaps with the COMPASS range, but the $x$ binning is different [4]. We have combined bins 2 and 3 , and bins 5 and 6 of HERMES, reducing the original 7 HERMES points into 5 points which are centered with good approximation in our bins 5, 6, 7, 8, and 9. The HERMES data have also been corrected by $D_{N N}$ while the effect of the cut $z<0.7$ and of the difference in energy have been neglected. A weighted mean of HERMES and COMPASS data has then been done, and the transversity functions have been extracted point by point as if all the data would have been taken by COMPASS. The overall situation is shown in Fig. 4.

The extracted values of $x h_{1}^{u_{v}}$ and $x h_{1}^{d_{v}}$ when using the 2021 projections for the deuteron are shown in Fig. 5, together with the $68 \%$ and $90 \%$ confidence bands obtained from 80000 replicas (right panel), and compared with the corresponding quantities obtained with the existing deuteron data (left panel).

As in Ref. [19] we have used the Collins asymmetries for positive and negative hadrons from the 2010 proton data and all the deuteron data assuming all the charged hadrons to be pions, while identified pions are about $70 \%$ of the "all hadron" sample. Using the identified pion asymmetries, the statistical uncertainty would increase by about $20 \%$.

Since $x h_{1}^{u_{v}}$ and $x h_{1}^{d_{v}}$ are obtained as linear functions of the four measured asymmetries (see Ref. [19]) their estimated values are correlated. Table 1 gives the correlation coefficients in the $9 x$ bins, both for the existing deuteron data and when using the projections of the proposed deuteron run. The correlation coefficients strongly depend on the relative statistics between the proton and 


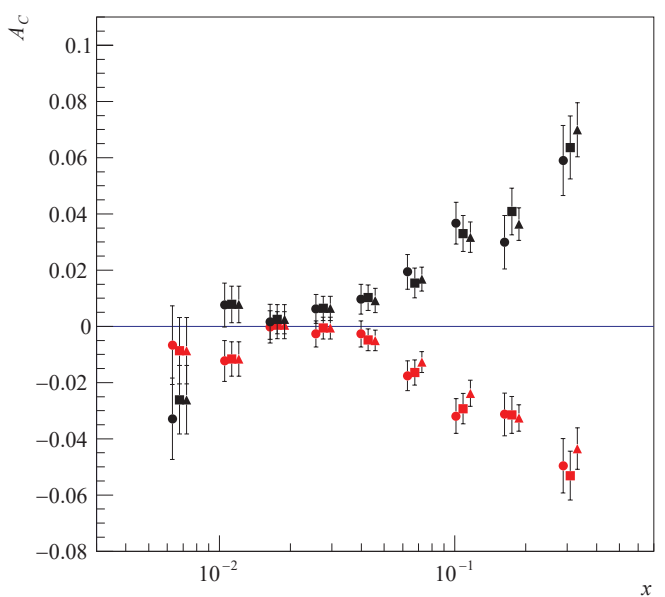

Figure 4: The Collins asymmetry for positive (red) and negative (black) hadrons from the existing proton data. In each $x$ bin, the first point (left to right) is from the 2010 COMPASS run, the second point is from the combined 2007 and 2010 COMPASS data, the third is obtained by adding also the HERMES data.

\begin{tabular}{|l|r|r|}
\hline$x$-bin & \multicolumn{2}{|c|}{$\rho\left(x h_{1}^{u_{v}}, x h_{1}^{d_{v}}\right)$} \\
\hline & present & projected \\
\hline $0.003-0.008$ & 0.494 & -0.029 \\
$0.008-0.013$ & 0.507 & -0.030 \\
$0.013-0.020$ & 0.509 & -0.042 \\
$0.020-0.032$ & 0.553 & -0.049 \\
$0.032-0.050$ & 0.641 & -0.009 \\
$0.050-0.080$ & 0.743 & 0.099 \\
$0.080-0.130$ & 0.764 & 0.033 \\
$0.130-0.210$ & 0.867 & 0.142 \\
$0.210-0.70$ & 0.863 & -0.100 \\
\hline
\end{tabular}

Table 1: Correlation coefficient between $x h_{1}^{u_{v}}$ and $x h_{1}^{d_{v}}$ in the different $x$ bins from numerical calculations, using the HERMES data, the 2007 and 2010 COMPASS proton data and present and projected deuteron errors.

the deuteron data. In the existing situation, which is largely unbalanced in favour of the protons, they are very close to 1 , while they are close to zero with the new deuteron run.

\section{Projections for the tensor charge uncertainties}

To evaluate the tensor charge the transversity PDFs for the valence quarks have to be integrated over $x$ from 0 to 1 . To avoid any possible bias, we prefer not to use any parametric function for the transversity PDFs, and simply integrate numerically the measured valence quark transversity values obtained as described in the previous section. The statistical uncertainty is then evaluated by adding up the errors of the measurements in the various $x$-bins. 

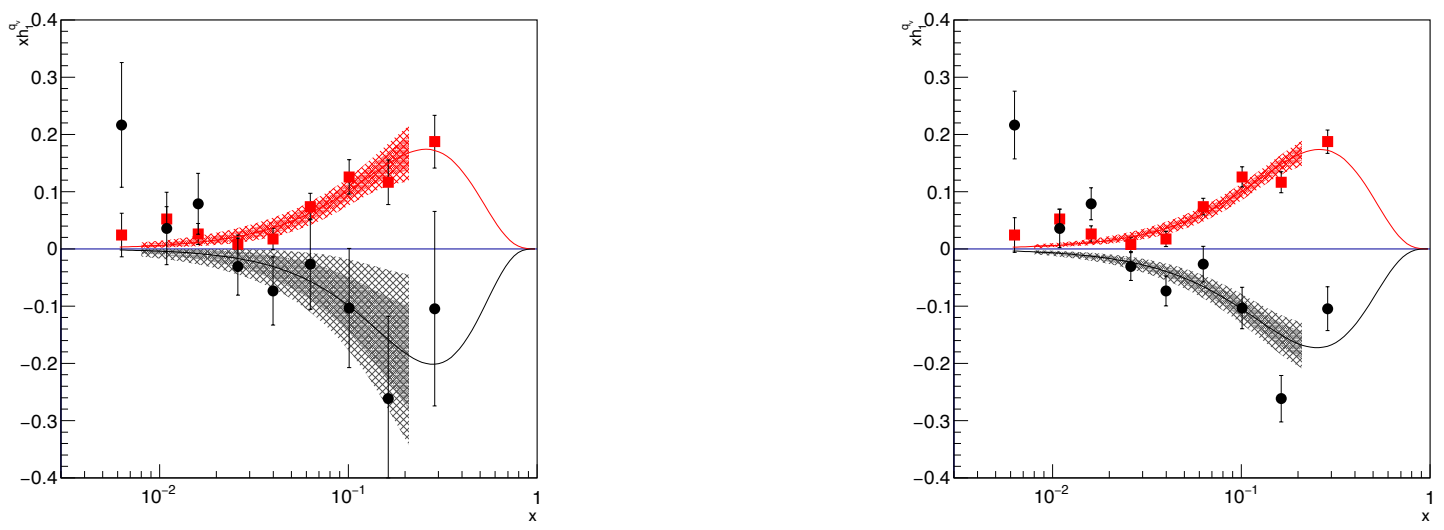

Figure 5: The points are the values for $x h_{1}^{u_{v}}$ (red squares) and $x h_{1}^{d_{v}}$ (black circles) extracted from the measured asymmetries. Left plot: existing deuteron data uncertainties. Right plot: projected uncertainties for the future deuteron run. Also shown, for both cases, are the $68 \%$ and $90 \%$ confidence bands obtained from 80000 replicas.

\begin{tabular}{|l|c|c|c|}
\hline & $\delta u=\int d x h_{1}^{u_{v}}(x)$ & $\delta d=\int d x h_{1}^{d_{v}}(x)$ & $g_{T}=\delta u-\delta d$ \\
\hline present & $0.201 \pm 0.032$ & $-0.189 \pm 0.108$ & $0.390 \pm 0.087$ \\
projected & $0.201 \pm 0.019$ & $-0.189 \pm 0.040$ & $0.390 \pm 0.044$ \\
\hline
\end{tabular}

Table 2: Truncated tensor charges from all existing proton and deuteron data ("present") and with the projected uncertainties for the deuteron data ("projected"). The integration runs over the range $0.008<x<$ 0.21 .

It is clear that COMPASS can give an important and unique contribution in a limited $x$-range, and the evaluation of the tensor charge ultimately will rely on the measurements of many experiments. In particular the SoLID Collaboration [21] at JLab12 is expected to contribute with very accurate measurements in the valence region, for $0.1<x<0.7$, in the future.

COMPASS, on the other hand, can give an accurate estimate of the tensor charge in the range $0.008<x<0.21$. For different reasons, the first $(0.003<x<0.008)$ and the ninth $(0.21<x<$ $0.7) x$-bins give fairly large contributions to the uncertainty of the tensor charge, therefore in the following we integrate the transversity PDFs over the central seven bins, skipping the first and the last.

The values of $\delta u, \delta d$ and $g_{T}$ in the selected $x$ range are given in Table 2, both for the present uncertainties and using the projected errors for the deuteron. The correlation coefficients of Table 1 are properly taken into account in the calculation of the uncertainties of the tensor charges. The reduction of the uncertainties on the integral of the $d$-quark when the projected measurements are 
taken into account is considerable, from 0.108 to 0.040 . This is the most important outcome we expect from the new run we propose. However, also the knowledge of the $u$-quark transversity integral improves, from 0.032 to 0.019 . For the tensor charge $g_{T}$ the uncertainty goes from 0.087 to 0.044 . The gain is smaller than for the $d$-quark case, for two reasons. First, the weights of the $u$ and $d$ quarks in the tensor charge are the same, thus the gain for the tensor charge is a sort of mean of the gains of the two PDFs. Secondly, with the present data the statistics of the proton data is much larger than that of the deuteron data, and, as a consequence, the correlation coefficients between the $u$ - and the $d$-transversity is large and positive, making the variance of the tensor charge smaller than the quadratic sum of the variances of the $\delta u$ and $\delta d$. On the contrary, the correlation coefficient between $x h_{1}^{u_{v}}$ and $x h_{1}^{d_{v}}$ becomes small (and negative) when one adds the new deuteron run, making the statistics of proton and deuteron asymmetries similar, and the variance of the tensor charge is essentially the quadratic sum of the variances of $\delta u$ and $\delta d$. It has to be stressed however that a situation in which the extracted values of $x h_{1}^{u_{v}}$ and $x h_{1}^{d_{v}}$ are almost uncorrelated is preferable to the one when $x h_{1}^{d_{v}}$ is strongly correlated to $x h_{1}^{u_{v}}$, which is the case presently.

The impact of the proposed deuteron measurement on the statistical accuracy of the tensor charge has been evaluated also with two other methods, namely

1. fitting with "reasonable" functions the extracted values of $x h_{1}^{u_{v}}(x)$ and $x h_{1}^{d_{v}}(x)$, and then integrating the curves obtained in this way, and

2. using "replicas" of the measured asymmetries.

The results obtained with these two more methods have slightly smaller uncertainties, but we prefer to quote the ones obtained with the numerical integration which are fully unbiased.

To summarize, at large $x$, JLab12 will provide very accurate partial measurements for $g_{T}$. At smaller $x(0.008<x<0.21)$ the COMPASS data will provide a contribution to $g_{T}$ with an uncertainty of \pm 0.044 . Without the new deuteron data from COMPASS, the evaluation of the tensor charge from only the future high precision JLab data would be affected by the error of the integration between 0 and 0.1 , which is difficult to be ascertained, and the result will anyhow be model dependent. We expect that, with the new COMPASS deuteron data, the uncertainty of the extrapolated contribution of the integration from 0 to 0.003 will be much smaller, and the uncertainty of the partial integration of the COMPASS data will be the resulting uncertainty on the tensor charge $g_{T}$.

To summarize, the new COMPASS measurement on a transversely polarized deuteron target will allow to reduce the uncertainties on the $\mathrm{d}$ quark PDFs. This will be particularly important for the transversity PDF, which allows to access the tensor charge $g_{T}$. At small $x$ values $(0.008<$ $x<0.21)$ the COMPASS data will provide a contribution to the measurement of $g_{T}$. with an uncertainty of \pm 0.044 . At large $x$, JLab12 should provide very accurate partial measurements for $g_{T}$, but without the new deuteron data from COMPASS the evaluation of the tensor charge would be affected by the error of the integration between 0 and 0.1 , which is difficult to be ascertained, and the result will anyhow be model dependent. We expect that, with the new COMPASS deuteron data, the uncertainty of the extrapolated contribution of the integration over $x$ from 0 to 0.003 will be much smaller, and the uncertainty of the partial integration of the COMPASS data might be the resulting overall uncertainty on the tensor charge $g_{T}$. 


\section{References}

[1] V. Barone et al., Prog. Part. Nucl. Phys. 65 (2010) 267.

[2] C. A. Aidala et al., Rev. Mod. Phys. 85 (2013) 655.

[3] H. Avakian et al., Eur. Phys. J. A52 (2016) 150, [Erratum: Eur. Phys. J. A52 No.6 (2016) 165].

[4] HERMES Collaboration, A. Airapetian et al., Phys. Lett. B693 (2010) 11.

[5] HERMES Collaboration, A. Airapetian et al., Phys. Rev. Lett. 103 (2009) 152002.

[6] COMPASS Collaboration, E. S. Ageev et al., Nucl. Phys. B765 (2007) 31.

[7] COMPASS Collaboration, M. G. Alekseev et al., Phys. Lett. B692 (2010) 240.

[8] COMPASS Collaboration, C. Adolph et al., Phys. Lett. B717 (2012) 376.

[9] COMPASS Collaboration, C. Adolph et al., Phys.Lett. B717 (2012) 383.

[10] Belle Collaboration, R. Seidl et al., Phys. Rev. D78 (2008) 032011, [Erratum: Phys. Rev.D86,039905(2012)].

[11] Z.-B. Kang et al., Phys. Rev. D93 (2016) 014009.

[12] M. Anselmino et al., Phys. Rev. D92 (2015) 114023.

[13] Jefferson Lab Hall A, X. Qian et al., Phys. Rev. Lett. 107 (2011) 072003.

[14] Jefferson Lab Hall A, Y. X. Zhao et al., Phys. Rev. C90 (2014) 055201.

[15] J.-W. Chen et al., Nucl. Phys. B911 (2016) 246.

[16] T. Bhattacharya et al., Phys. Rev. D94 (2016) 054508.

[17] A. Courtoy et al., Phys. Rev. Lett. 115 (2015) 162001.

[18] COMPASS Collaboration, d-Quark Transversity and Proton Radius, Addendum to the COMPASS-II Proposal, CERN-SPSC-2017034 SPSC-P-340-ADD-1, April 5, 2018.

[19] A. Martin et al., Phys. Rev. D91 (2015) 014034.

[20] E. Maguire et al., J. Phys. Conf. Ser. 898 (2017) 102006.

[21] Z. Ye et al., Phys. Lett. B767 (2017) 91. 